\title{
Substance use and psychiatric disorders in patients referred to consultation-liaison psychiatry within a regional general hospital
}

Murray Tucker ( $\nabla$ tuckermurray8@gmail.com )

Barwon Health MHDAS https://orcid.org/0000-0001-8051-1959

Harry Hill

Barwon Health MHDAS

Emma Nicholson

Barwon Health MHDAS

Steven Moylan

Barwon Health MHDAS

\section{Research Article}

Keywords: consultation-liaison psychiatry, dual diagnosis, comorbidity, substance use disorders, mental disorders

Posted Date: June 17th, 2021

DOl: https://doi.org/10.21203/rs.3.rs-622635/v1

License: (9) This work is licensed under a Creative Commons Attribution 4.0 International License. Read Full License

Version of Record: A version of this preprint was published at International Journal of Mental Health and Addiction on July 2nd, 2021. See the published version at https://doi.org/10.1007/s11469-021-00576-1. 


\section{Abstract}

Little is known about clinically important differences between patients depending on the number of comorbid psychiatric disorders, or the presence or absence of a substance use disorder (SUD) comorbidity. This study investigated for differences in psychosocial disadvantage, psychiatric disorders, and health service amongst 194 general hospital patients referred to consultation-liaison psychiatry (CLP) with no psychiatric diagnosis, single psychiatric diagnosis, multiple (non-SUD) psychiatric diagnoses, or psychiatric diagnosis plus SUD comorbidity. The results showed that SUDs were the commonest diagnostic category (34\%). The SUD comorbidity group had more disadvantaged housing, were prescribed most psychoactive medications, and $20 \%$ prematurely self-discharged against medical advice. Increased SUDs were associated with reduced length of stay, men, younger age, increased investigations, and reduced private health insurance subscription. Patients with SUD comorbidity versus multiple psychiatric diagnosis had reduced odds of Adjustment Disorder, Somatic Symptom Disorder, and Insomnia Disorder. Post Traumatic Stress Disorder was the strongest predictor of multiple SUDs, followed by Cluster B personality disorders. In conclusion, SUDs have become a leading clinical focus for CLP. The presence or absence of SUDs amongst patients with multiple psychiatric disorders has important clinical implications for engagement, diagnosis, prescribing, and outpatient follow-up.

\section{Introduction}

Substance use disorder (SUD) related hospital presentations have become a leading health problem. Approximately $33 \%$ of people presenting to Australian hospitals have a SUD problem (Butler, Reeve, Viney, \& Burns, 2016), which represents a three-fold increase over the past decade (Tran et al., 2020). Whilst SUDs are increasingly common amongst general hospital inpatients, they are often under-detected or not referred for specialist assessment and management by consultation-liaison psychiatry (CLP) (Diehl et al., 2009; Smith, Clarke, \& Handrinos, 1995; Smothers, Yahr, \& Ruhl, 2004). SUDs are highly comorbid with psychiatric disorders (Buckley \& Brown, 2006), contribute to disengagement from medical treatment, and are associated with social disadvantage, medical complications, and increased health service utilization (Nordeck et al., 2018). Dedicated general hospital SUD liaison services have been developed to address these issues directly with the aim of reducing medical complications, healthcare costs, and rates of rehospitalization (Butler et al., 2016; Nordeck et al., 2018; Tadros et al., 2013).

Despite the extensive biopsychosocial problems associated with SUDs, few studies have examined the types, frequencies, and patterns of substance use amongst patients referred to CLP. Instead, studies have reported SUDs as a single diagnostic category or a single main psychiatric diagnosis rather than the patterns of comorbidity (Bourgeois, Wegelin, Servis, \& Hales, 2005; Chandarana, Conlon, \& Steinberg, 1988; Devasagayam \& Clarke, 2016; Dilts, Mann, \& Dilts, 2003; Lipowski \& Wolston, 1981; Rothenhausler, Ehrentraut, \& Kapfhammer, 2001). Previous CLP studies across different countries and hospital settings have found that the most common disorders of CLP practice have been Major Depression, Adjustment Disorder, and Delirium (Bourgeois et al., 2005; Clarke \& Smith, 1995; Holmes, Handrinos, Theologus, \& Salzberg, 2011; Huyse et al., 2001; Smith, Clarke, \& Herrman, 1993; Wand, Wood, Macfarlane, \& Hunt, 
2016). The frequencies of SUDs were 1.5-12\% (Clarke \& Smith, 1995; Diefenbacher \& Strain, 2002; Holmes et al., 2011; Krautgartner, Alexandrowicz, Benda, \& Wancata, 2006; Loewenstein \& Sharfstein, 1983; McKegney, McMahon, \& King, 1983; Smith et al., 1993). However, these low rates of SUDs now appear inconsistent with the changing landscape of CLP practice towards a more contemporary emphasis on SUDs, which was the leading diagnostic category in two recent studies (Desai, Shah, Shah, Sharma, \& Zankat, 2016; Lyne et al., 2010). Patters of multiple substance use are also infrequently examined amongst general hospital patients. However, multiple substance use may be linked with particular psychopathology, personality disorders, and trauma (Donald, Arunogiri, \& Lubman, 2019; Khantzian, 1997; Lubman, Hall, Pennay, \& Rao, 2011; Mills, Teesson, Ross, \& Peters, 2006; Nesse \& Berridge, 1997; Pagura et al., 2010).

Multiplicity of mental disorders is also an emerging measure of assessment and management complexity for patients within the CLP setting (Bourgeois et al., 2005). At least $25 \%$ of patients referred to CLP have multiple mental disorders (Bourgeois et al., 2005; Holmes et al., 2011; Loewenstein \& Sharfstein, 1983; McKegney et al., 1983; Wood, Wand, \& Hunt, 2015; Zimmerman \& Mattia, 1999). However, there has been little investigation as to whether this multiplicity measure represents a different diagnostic construct for patients with or without SUD comorbidity. Patients who meet the criteria for at least one SUD and psychiatric disorder simultaneously are often defined in the psychiatric literature as having a 'substance use comorbidity' or 'dual diagnosis'. Individuals with multiplicity of psychiatric disorders versus single or no psychiatric disorder may have similar problems to individuals with psychiatric disorder and SUD comorbidity, such as greater psychosocial impairment, treatment resistance, medical comorbidity, and rehospitalization (AlHW, 2012; Bourgeois et al., 2005; Wancata, Benda, Windhaber, \& Nowotny, 2001; Zimmerman \& Mattia, 1999). However, some studies lacked a clear definition whether the multiplicity of disorders measure involves the presence or absence of SUDs (Bourgeois et al., 2005). Hence, it is unclear whether the problems associated with multiple mental disorders are from comorbid SUDs or not.

The aim of the current study was to examine for differences in demographics, physical health, psychiatric variables, and health service utilization based on the number of diagnosed psychiatric disorders and the presence or absence of SUD comorbidity in a cohort of general hospital inpatients referred to CLP. It was also of interest to characterize the types, frequencies, and patterns of comorbidity between psychiatric disorders and SUDs.

\section{Methods}

\section{Study Design and Setting}

The study was retrospectively performed and involved all patients referred to the CLP unit over 3-months from February 2018 to May 2018. Medical records were re-checked at 6-months post discharge to measure the number of rehospitalizations. The setting was a large regional tertiary public hospital with 437 acute general hospital beds in Victoria, Australia. The CLP unit covered all forms of mental health 
and drug and alcohol consultations and operated separately from the emergency department. Ethical clearance was obtained from the Barwon Health Research Ethics, Governance, and Integrity Unit (reference 18/25).

\section{Data Collection}

Inclusion criteria were general hospital inpatients who were referred to CLP via an electronic typed referral which was stored in a clinical database. Any patients referred by telephone also required a typed referral. Exclusion criteria were patients who were referred to CLP but were discharged prior to psychiatric assessment or who died during their admission. All data were extracted from electronic medical records and recorded de-identified into electronic spreadsheets by the primary author. Specific reference was made to demographic forms, emergency department and ambulance records, the typed referral to CLP, progress notes by all clinical staff, medication charts, and discharge summaries.

Psychiatric diagnoses were derived from the documentation of either a college-accredited psychiatric trainee (registrar) or consultant psychiatrist following at least one face-to-face psychiatric assessment. SUDs were derived from the patient's self-reported use documented by either CLP or the referring specialty. If multiple SUDs were present for a single substance (e.g. intoxication, withdrawal, dependence), only one SUD diagnosis was recorded. To determine inter-rater reliability, the psychiatric diagnoses of 25 randomly selected patients were compared between their inpatient CLP assessment and a post-discharge outpatient assessment by a consultant psychiatrist. The percent agreement was $84.4 \%$. Psychiatric diagnoses and SUDs were categorized using the Diagnostic and Statistical Manual of Mental Disorders version 5 (DSM-5, American Psychiatric Association, 2013).

\section{Data Analysis}

Outcome measures were demographic indicators (i.e. age, sex, singleness, indigenous status, private health insurance status, government welfare, not in training/study/employment, and no fixed abode or commission housing), psychiatric history variables (i.e. documented past psychiatric history, current case management, chronic pain history, electroconvulsive therapy history, and long acting injectable antipsychotic history), CLP referral and consultation variables (i.e. numbers of referral reasons, CLP reviews, CLP recommendations, and length of stay), diagnostic variables (e.g. DSM-5 psychiatric disorder and SUD), and clinical variables (e.g. numbers of all medications, psychiatric medications, medical diagnoses, investigations, and hospital readmissions).

Length of stay (LOS) was calculated as the time difference between admission and discharge. Reasons for CLP referral were categorized and summed for each patient using a classification system described by Smith and colleagues (1993) (e.g. diagnostic clarification, risk assessment, etc.). Frequencies of each DSM-5 psychiatric disorder and SUD were determined. Frequencies of paired comorbidities between (a) psychiatric disorders and (b) SUDs were determined for individual patients, summed across the cohort, and then displayed in bubble plots to reveal the prominent patterns of comorbidity. Data were processed using custom software (R2015a, MathWorks). 


\section{Statistical Analysis}

Patients were divided into four groups: (a) no psychiatric diagnosis and no SUD diagnosis (no diagnosis status), (b) single psychiatric diagnosis excluding SUDs (single diagnosis status), (c) multiple psychiatric diagnoses excluding SUDs (multiple diagnosis status), and (d) one or more psychiatric diagnosis plus one or more comorbid SUDs (SUD comorbidity status). Fisher's tests examined for group differences in dichotomous demographic variables. Group differences in the other dependent variables were analyzed with one-way Multivariate Analysis of Covariance (MANCOVA) with age as a covariate. In the event of a significant MANCOVA, post-hoc univariate F-tests examined for specific group differences.

Stepwise multiple regression analyses examined associations of (a) number of psychiatric diagnoses or (b) number of SUDs with demographic, psychiatric history, CLP referral and consultation, and clinical variables. Forward stepwise multivariate logistic regression with odds ratios (OR) determined the significant and independent predictors for patients with (a) multiple diagnosis versus SUD comorbidity status, and (b) non-multiple SUD versus multiple SUD status. Potential predictor variables for this analysis were age, sex, and DSM- 5 psychiatric diagnosis. Statistical analysis was performed using the Statistics Package for Social Sciences (SPSS, version 25) with significance accepted at $P<0.05$.

\section{Results}

\section{Frequencies of psychiatric diagnoses}

In total 461 diagnoses in 194 patients were recorded by CLP over the three-month study period (Fig. 1). The most common diagnostic category was substance and addictive disorders (156 diagnoses). Prominent SUDs were tobacco (43\%), alcohol (21\%), cannabis (14\%), amphetamines (12\%), opioids (8\%), and benzodiazepines (2\%), and one person had a gambling disorder. Common psychiatric diagnoses were Post Traumatic Stress Disorder (PTSD, $n=29)$, Borderline Personality Disorder (BPD, $n=27)$, Major Depressive Disorder (MDD, $n=18)$, Adjustment disorder $(n=17)$, Delirium $(n=13)$, and Generalised Anxiety Disorder (GAD, $n=11)$. Depression was the most suspected diagnosis by referrers ( 55 referrals), but this was only confirmed by the CLP assessment in $25 \%$ of cases.

\section{Group differences in outcome measures}

Patients with SUD comorbidity were most often referred to CLP (38.7\%), followed by those with multiple psychiatric diagnoses (21.6\%), a single psychiatric diagnosis (18\%), and no diagnosis $(11.9 \%)$.

Additionally, there were twelve patients with a single SUD only (6.2\%) and seven patients diagnosed with multiple SUDs and no comorbid psychiatric disorder (3.6\%). Significant group differences were detected in the demographic, pasty history, referral and consultation, and clinical and diagnostic variables (Table 1). 
Table 1

Demographic, psychiatric history, and clinical variables for groups of the referred patients.

\begin{tabular}{|c|c|c|c|c|c|}
\hline Variable & $\begin{array}{l}\text { No } \\
\text { diagnosis }\end{array}$ & $\begin{array}{l}\text { Single } \\
\text { diagnosis }\end{array}$ & $\begin{array}{l}\text { Multiple } \\
\text { diagnosis }\end{array}$ & $\begin{array}{l}\text { SUD } \\
\text { comorbidity }\end{array}$ & Test statistics \\
\hline $\begin{array}{l}\text { Demographic Variables } \\
(\mathrm{N}, \%)\end{array}$ & & & & & $\begin{array}{l}\text { F test for age \& } \\
\text { Fisher's tests }\end{array}$ \\
\hline $\mathrm{N}$ & 23 & 35 & 42 & 75 & \\
\hline Age & $45 \pm 25$ & $53 \pm 23$ & $48 \pm 24$ & $43 \pm 16$ & $F=2.10, P=0.10$ \\
\hline \multirow[t]{2}{*}{ Sex } & M (39) & M (46) & $M(36)$ & M (41) & $D=0.86 \mathrm{P}=0.83$ \\
\hline & $\mathrm{F}(61)$ & $F(54)$ & $F(64)$ & $F(59)$ & \\
\hline Single & $12(52)$ & $19(54)$ & $30(71)$ & $54(72)$ & $D=5.76, \mathrm{P}=0.13$ \\
\hline ATSI & $1(4)$ & $1(3)$ & $0(0)$ & $4(5)$ & $D=2.37, \mathrm{P}=0.50$ \\
\hline $\begin{array}{l}\text { Private Health } \\
\text { Insurance }\end{array}$ & $8(35)$ & $10(29)$ & $9(21)$ & $8(11) \star \nabla$ & $D=9.20, \mathrm{P}=0.02$ \\
\hline Government Pension & $15(65)$ & $16(46)$ & $30(71) \rrbracket$ & $62(83) \star \nabla$ & $\begin{array}{l}D=15.46, P< \\
0.001\end{array}$ \\
\hline Disadvantaged housing & $3(14)$ & $3(9)$ & $7(17)$ & $23(36) \star \nabla \psi$ & $\begin{array}{l}D=10.63, \mathrm{P}= \\
0.01\end{array}$ \\
\hline NEET & $15(71)$ & $23(70)$ & $32(76)$ & $59(83)$ & $D=3.11, \mathrm{P}=0.37$ \\
\hline $\begin{array}{l}\text { Psychiatric History } \\
\text { Variables }\end{array}$ & & & & & Fisher's Tests \\
\hline Past psychiatric history & $12(55)$ & $24(69)$ & $39(93) * \nabla$ & $67(89) \star \nabla$ & $\begin{array}{l}D=19.37, \mathrm{P}< \\
0.001\end{array}$ \\
\hline Case management & $0(0)$ & $6(17)$ & $12(29) \star$ & $14(19) *$ & $D=9.22, \mathrm{P}=0.02$ \\
\hline Chronic pain & $9(41)$ & $8(23)$ & $12(29)$ & $23(31)$ & $D=2.15, \mathrm{P}=0.55$ \\
\hline ECT history & $0(0)$ & $0(0)$ & $5(12)$ & $3(4)$ & $D=5.79, \mathrm{P}=0.07$ \\
\hline $\begin{array}{l}\text { LAl antipsychotic } \\
\text { history }\end{array}$ & $0(0)$ & $0(0)$ & $2(5)$ & $6(8)$ & $D=3.58, \mathrm{P}=0.27$ \\
\hline
\end{tabular}

Values are mean \pm standard deviation for continuous variables or frequency counts with percentage in parentheses for nominal variables. *Significantly different from the no diagnosis group $(P<0.05)$; 《 Significantly different from the single diagnosis group $(P<0.05) ; \psi$ Significant difference between the multiple diagnosis group and SUD comorbidity group $(P<0.05)$. ATSI = Aboriginal or Torres Strait Islander; $\mathrm{CLP}=$ consultation-liaison psychiatry; $D=$ test statistic for Fisher-Freeman-Halton test; $E C T=$ electroconvulsive therapy; $\mathrm{F}=$ female; $\mathrm{M}=$ male; $\mathrm{LAI}=$ long-acting injectable; MANCOVA = multivariate analysis of covariance; NEET = not in employment, education, or training. Disadvantaged housing = government housing or no fixed abode. 


\begin{tabular}{|c|c|c|c|c|c|}
\hline Variable & $\begin{array}{l}\text { No } \\
\text { diagnosis }\end{array}$ & $\begin{array}{l}\text { Single } \\
\text { diagnosis }\end{array}$ & $\begin{array}{l}\text { Multiple } \\
\text { diagnosis }\end{array}$ & $\begin{array}{l}\text { SUD } \\
\text { comorbidity }\end{array}$ & Test statistics \\
\hline $\begin{array}{l}\text { Referral \& Consultation } \\
\text { Variables }\end{array}$ & & & & & $\begin{array}{l}\text { MANCOVA } \mathrm{P}< \\
0.001\end{array}$ \\
\hline Referral reasons & $2.3 \pm 1.3$ & $2.1 \pm 1.1$ & $2.7 \pm 1.7$ & $2.9 \pm 1.8 \otimes$ & $F=2.77, P=0.04$ \\
\hline CLP reviews & $1.1 \pm 0.4$ & $2.2 \pm 3.3$ & $\begin{array}{l}5.6 \pm \\
14.4^{\star}\end{array}$ & $2.1 \pm 3.3 \psi$ & $F=2.88, P=0.04$ \\
\hline CLP recommendations & $1.6 \pm 1.3$ & $3.2 \pm 1.8^{\star}$ & $\begin{array}{l}4.3 \pm \\
2.8^{\star} \rrbracket\end{array}$ & $3.7 \pm 2.0 *$ & $F=8.64, P<0.001$ \\
\hline Length of stay (days) & $9.1 \pm 11.5$ & $15.3 \pm 30.0$ & $\begin{array}{l}14.0 \pm \\
18.0\end{array}$ & $8.1 \pm 12.2$ & $\begin{array}{l}F=1.37, P= \\
0.253\end{array}$ \\
\hline $\begin{array}{l}\text { Diagnostic \& Clinical } \\
\text { Variables }\end{array}$ & & & & & $\begin{array}{l}\text { MANCOVA } \mathrm{P}< \\
0.001\end{array}$ \\
\hline All medications & $\begin{array}{l}15.3 \pm \\
10.2\end{array}$ & $12.5 \pm 6.2$ & $14.3 \pm 9.7$ & $14.6 \pm 8.6$ & $F=2.51, P=0.06$ \\
\hline $\begin{array}{l}\text { Psychiatric } \\
\text { medications }\end{array}$ & $1.3 \pm 1.1$ & $2.0 \pm 1.8$ & $2.2 \pm 1.8^{\star}$ & $3.1 \pm 1.8 * \nabla \Psi$ & $F=8.76, P<0.001$ \\
\hline Psychiatric diagnoses & $0.0 \pm 0.0$ & $1.0 \pm 0.0^{*}$ & $\begin{array}{l}2.7 \pm \\
1.1 \star \mathbb{\nabla}\end{array}$ & $2.0 \pm 1.1 * \nabla \psi$ & $F=54.0, P<0.001$ \\
\hline $\begin{array}{l}\text { Substance Use } \\
\text { Disorders }\end{array}$ & $0.0 \pm 0.0$ & $0.0 \pm 0.0$ & $0.0 \pm 0.0$ & $1.7 \pm 0.9 * \nabla \Psi$ & $\begin{array}{l}F=127.0, P< \\
0.001\end{array}$ \\
\hline Medical diagnoses & $6.2 \pm 6.1$ & $5.6 \pm 3.9$ & $6.5 \pm 5.0$ & $5.7 \pm 4.1$ & $F=1.55, P=0.20$ \\
\hline Investigations & $35 \pm 45$ & $33 \pm 46$ & $44 \pm 57$ & $44 \pm 94$ & $F=0.56, P=0.64$ \\
\hline Hospital re-admissions & $0.7 \pm 1.1$ & $1.1 \pm 1.7$ & $1.0 \pm 1.3$ & $1.3 \pm 2.1$ & $F=0.93, P=0.43$ \\
\hline \multicolumn{6}{|c|}{$\begin{array}{l}\text { Values are mean } \pm \text { standard deviation for continuous variables or frequency counts with percentage } \\
\text { in parentheses for nominal variables. *Significantly different from the no diagnosis group }(P<0.05) ; \otimes \\
\text { Significantly different from the single diagnosis group }(\mathrm{P}<0.05) ; \Psi \text { Significant difference between the } \\
\text { multiple diagnosis group and SUD comorbidity group }(P<0.05) \text {. ATS = Aboriginal or Torres Strait } \\
\text { Islander; } C L P=\text { consultation-liaison psychiatry; } D=\text { test statistic for Fisher-Freeman-Halton test; ECT = } \\
\text { electroconvulsive therapy; } F=\text { female; } M=\text { male; } L A I=\text { long-acting injectable; MANCOVA = multivariate } \\
\text { analysis of covariance; NEET = not in employment, education, or training. Disadvantaged housing = } \\
\text { government housing or no fixed abode. }\end{array}$} \\
\hline
\end{tabular}


Table 2

Significant predictors of group status in the forward stepwise logisitic regression analyses

Multiple Psychiatric Diagnosis vs. SUD comorbidity

Predictor Variable

Adjustment Disorder

Somatic Symptom

Disorder

Insomnia Disorder

Odds

Ratio

0.16

0.06

0.19

$P=0.027$

$P=0.012$

$P=0.012$
PTSD

ASPD

ASPD = antisocial personality disorder; PTSD = post traumatic stress disorder; $S U D=$ substance use disorder. The odds ratios reference the latter group in each analysis. E.g. Odds ratios $>1$ indicate increased odds of the predictor variables in the latter group versus the former group.

For the demographic variables, individuals from the SUD comorbidity group were significantly less likely to have private health insurance compared with the no diagnosis and single diagnosis groups $\left(P^{\prime} s<0.05\right)$ and significantly more likely to have disadvantaged housing compared to all other groups $\left(P^{\prime} s<0.05\right)$. There was a significantly higher proportion of individuals with a government pension in the multiple diagnosis group compared with the single diagnosis group $(P<0.05)$, and for the SUD comorbidity group compared with the no diagnosis and single diagnosis groups $\left(P^{\prime} s<0.05\right)$.

For the past psychiatric history variables, individuals from the multiple diagnosis and SUD comorbidity groups were significantly more likely to have an existing past psychiatric history compared with the no diagnosis and single diagnosis groups ( $P$ 's $<0.05$ ). The multiple diagnosis group and SUD comorbidity group had significantly higher proportion of individuals who had public sector psychiatric case management compared with the no diagnosis group ( $P$ 's $<0.05)$.

The MANCOVA was significant for the referral and consultation variables $\left(F_{12,442}=3.41\right.$, Wilks' Lambda $=$ $0.79, P<0.001)$. Referrers had significantly more reasons to request CLP review for the SUD comorbidity group compared with the single psychiatric diagnosis group $(P<0.05)$. The multiple psychiatric diagnosis group required significantly more CLP reviews compared to the no diagnosis and SUD comorbidity groups ( $P$ 's $<0.05$ ). The no diagnosis group had significantly less management recommendations compared with the other groups $\left(P^{\prime} \mathrm{s}<0.05\right)$, and the multiple psychiatric diagnosis group had significantly more management recommendations compared with the single psychiatric diagnosis group $(P<0.05)$.

The MANCOVA was significant for the diagnostic and clinical variables $\left(F_{21,460}=21.8\right.$, Wilks' Lambda $=$ $0.13, P<0.001)$. The SUD comorbidity group were prescribed significantly more psychiatric medications and had significantly more SUDs compared with all other groups ( $P$ 's $<0.05)$. The SUD comorbidity group also had significantly more psychiatric diagnoses compared with the no diagnosis and single diagnosis groups ( $P$ 's $<0.001)$. The multiple diagnosis group was prescribed significantly more psychiatric 
medications compared with the no diagnosis group $(P<0.05)$ and had significantly more psychiatric diagnoses compared with all other groups (P's<0.001).

\section{Multiple regression analyses}

Multiple regression analysis was significant for number of psychiatric diagnoses $\left(R=0.57, F_{3,169}=27.0\right.$, $P<0.001)$. The significant predictor variables were number of CLP management recommendations $(\beta=$ $0.42)$, the presence of past psychiatric history $(\beta=0.26)$, and government pension status $(\beta=0.21)$.

Multiple regression analysis was also significant for number of SUDs $\left(R=0.46, F_{7,165}=6.4, P<0.001\right)$. The significant predictor variables were number of referral reasons $(\beta=0.14)$, length of stay $(\beta=-0.31)$, number of inpatient psychiatric medications $(\beta=0.28)$, private health insurance status $(\beta=-0.15)$, age $(\beta$ $=-0.20)$, male sex $(\beta=-0.17)$, and number of investigations $(\beta=0.22)$.

\section{Co-occurrence of psychiatric diagnoses and substance use disorders}

A bubble plot of the frequencies at which psychiatric diagnoses co-occurred, depending on level of substance use, is displayed in Fig. 2. Increased level of substance use was associated with trauma/stressor disorders, anxiety disorders, and personality disorders (i.e. red bubbles along the corresponding single diagnostic axis). Cluster B personality disorders (primarily Borderline) mostly cooccurred with trauma/stressor disorders (primarily PTSD), and this co-occurrence was more frequent in those with increased substance use (i.e. increasing size of blue and red bubbles respectively at the intersection of PTSD and Cluster B diagnostic categories). Patients with no substance use often had neurodevelopmental disorders (e.g. ASD and ID).

The patterns of combined substance use for patients with multiple SUDs is displayed in Fig. 3. Common combinations were tobacco and alcohol, tobacco and benzodiazepines, and benzodiazepines and opioids. Those with MDD had no clear preferential pattern (i.e. similarly sized red bubbles across multiple substance combinations). Patterns of substance use in BPD and PTSD were similar (i.e. red and black bubbles are similar in size and distribution). Those with GAD preferentially used benzodiazepines in combination with alcohol or tobacco, whereas those with PTSD and BPD used benzodiazepines in combination with opioids or tobacco.

\section{Logistic regression analyses}

Logistic regression analysis was significant for the multiple psychiatric diagnoses group versus the SUD comorbidity group (Chi Sq $=22.1, P<0.001$, Nagelkerke $R^{2}=0.236$, classification accuracy $74 \%$ ) and for patients with or without multiple SUDs (Chi Sq $=23.4, P<0.001$, Nagelkerke $R^{2}=0.17$, classification accuracy $79 \%$ ). Significant predictor variables for both analyses are displayed in Table 3.

\section{Discussion}


The 461 recorded CLP diagnoses reflected high levels of psychiatric impairment within this cohort with SUDs representing the largest burden of illness (34\%). Patients with SUD comorbidity were most often referred to CLP (39\%) and their referrals contained more issues of concern. These findings are consistent with the strong demand for CLP services for substance use associated problems in general hospital settings (Butler et al., 2016; Nordeck et al., 2018). From a psychiatric epidemiology perspective, our results support other studies which have found that SUDs can be the leading management issue for general hospital CLP services, above that of Major Depression, Adjustment disorder, and Delirium (Desai et al., 2016; Lyne et al., 2010). Patients with multiple psychiatric diagnoses represented $22 \%$ of the cohort, which is in agreement with the $25 \%$ previously reported (Bourgeois et al., 2005; Holmes et al., 2011; Loewenstein \& Sharfstein, 1983; McKegney et al., 1983; Wood et al., 2015; Zimmerman \& Mattia, 1999).

Patients with more mental disorders, either with or without SUD comorbidity, had multiple markers of social disadvantage, increased psychiatric case management, and required more intensive CLP involvement. These findings are supportive that a multiplicity of mental disorders can be a useful marker of psychiatric complexity and health service utilization (Bourgeois et al., 2005). However, there were also some important differences between the multiple diagnosis and SUD comorbidity groups which indicates they are not interchangeable entities on face value despite some previous studies including SUDs within the multiplicity of diagnosis concept. Patients with SUD comorbidity had the highest rates of disadvantaged housing, were prescribed the most psychotropic medications, and had significantly reduced odds of comorbid Adjustment Disorder, Somatic Symptom Disorder, or Insomnia Disorder compared with the multiple diagnosis group. The significant associations between increased number of SUDs and males, younger age, PTSD, and Cluster B Personality Disorders replicates findings from other settings such as community samples and addiction treatment centers (Mills et al., 2006; Regier et al., 1990; Trull et al., 2018; Wapp et al., 2015).

Reduced association between somatic symptom disorders and substance use has been reported in a large US community-based cohort study (Simon \& VonKorff, 1991). A potential explanation is that unpleasant somatic side effects of substances could exacerbate symptoms in those with somatic symptom disorder. Substance users may have also complained less about insomnia due to sedating effects of intoxication/withdrawal or sedating prescription medications. Common prescribing practices for patients with SUDs were pro re nata (PRN) for acute withdrawal symptoms or for psychological distress, usually with PRN medication discontinuation prior to discharge. More frequent prescribing for these patients may also coincide with greater acceptability amongst active substance users to rely upon exogenous substances to regulate negative affective states (Nesse \& Berridge, 1997).

The SUD comorbidity group had reduced number of CLP reviews versus the multiple diagnosis group, a non-significant trend towards the shortest LOS, and shortest LOS was most strongly associated with increased numbers of SUDs. A post-hoc analysis revealed that $20 \%$ of individuals with SUD comorbidity self-discharged 'against medical advice' prior to satisfactory completion of their hospital treatment. These patients averaged a substantially shorter LOS of $4.3 \pm 4.7$ days. This finding is suggestive of 
problems with therapeutic alliance in patients with SUD comorbidity, resulting in premature discharge possibly to self-manage cravings or other psychosocial stressors.

Substance use and trauma commonly feature in the histories of patients with BPD (Pagura et al., 2010; Trull et al., 2018; Wapp et al., 2015). Our results revealed that BPD-PTSD was the commonest psychiatric comorbidity across all potential couplings of DSM- 5 diagnoses, and that the likelihood of BPD-PSTD comorbidity was increased in patients with heavier substance use. Does this association between BPD, PTSD, and SUDs represent a three-way interaction of independent factors, or an overlap of shared diagnostic criteria? The DSM-5 indicates that individuals with PTSD or BPD may both engage in reckless or self-destructive behaviors such as excessive drug or alcohol use (Donald et al., 2019). Whilst patients with PTSD-BPD comorbidity might use more substances to self-manage their greater burden of psychiatric symptoms (Trull et al., 2018), it is also possible that increased substance use exposes individuals to traumatic experiences which increases their risk of PTSD (Mills et al., 2006). BPD has also been conceptualized as the outcome of a chronic form of PTSD (Pagura et al., 2010; Trull et al., 2018), which could bias diagnostic practices in favor of BPD-PTSD comorbidity. When BPD was tested independently from PTSD in the logistic regression analysis, BPD was not a significant predictor of patients with multiple substance use. Whereas Antisocial Personality Disorder, which had no observed comorbidity with PTSD, captured significantly unique information about multiple substance use that led to its inclusion in the model alongside PTSD. Taken together, our findings suggest that PTSD is most strongly linked with multiple substance use compared to Cluster B personality disorders.

Tobacco and alcohol were the most used substances in the cohort, possibly reflecting their higher availability in the community compared with illicit substances. Preferential coupling between types of SUDs and psychiatric disorders was suggestive of targeted use based on psychoactive properties. For example, patients with GAD preferentially combined substances with acute anxiolytic effects such as alcohol, prescribed or illicit benzodiazepines, and tobacco. They also avoided amphetamine type stimulants which could exacerbate anxiety. Patients with PTSD or BPD combined opioids, benzodiazepines, and tobacco. Potentially the anxiolytic and euphoric properties of these substances may have counteracted the anhedonic and dysphoric symptoms common to PTSD and BPD disorders (Mills et al., 2006; Trull et al., 2018). Whilst these findings may lend some support to the self-medication hypothesis for substance use (Khantzian, 1997), the challenge in establishing clear etiological timelines of psychiatric symptoms versus substance use cross-sectionally suggests that substance-induced psychiatric symptoms could also account for the comorbidity patterns. The broad range of substance use across the cohort implies that accessibility and affordability were possibly other factors influencing use.

\section{Strengths and limitations}

A limitation of the study was its retrospective design. However, an advantage of the design was absence of the Hawthorne effect. Clinician bias may have influenced diagnostic practices and the observed patterns of comorbidity. For example, the lower rates of depressive disorders compared with previous

Page $11 / 18$ 
studies may also reflect diagnostic bias towards adjustment disorder with depressed mood, or attribution of depressive symptoms to substance use or medical illness. Additionally, CLP clinicians may only document the psychiatric disorders that are relevant to the patient's presentation or the specific referral questions. This may explain the absence of some disorders like gender dysphoria, elimination disorders, and dissociative disorders from the results. Structured clinical interviews in prospective studies can increase diagnostic accuracy. However, the psychiatric diagnoses in this study were derived from the usual clinical process, which arguably improves the relevance of the results for routine clinical practice. The absence of substance-induced disorders reflects the study's general hospital setting. For example, patients with substance induced psychotic disorders were assertively admitted to the psychiatric ward from the emergency department which bypassed the CLP service.

\section{Implications for practice and service planning}

Our findings highlight that assessing and managing SUDs is a key practice area in CLP. Patients with SUD comorbidity may experience difficulties in stable housing and engaging in treatment within busy general hospital settings despite support from CLP services. There is emerging evidence that dedicated inpatient drug and alcohol consultation services are a cost-effective intervention that improves engagement in treatment, and significantly reduces emergency presentations and hospital readmissions (Butler et al., 2016; Nordeck et al., 2018). Therefore, a dedicated multidisciplinary drug and alcohol liaison attachment within the CLP service structure could potentially enhance this aspect of service delivery within general hospitals where SUDs predominate. Patterns of comorbidity between SUDs and psychiatric disorders may serve as a useful prompt for CLP clinicians and students to explore during psychiatric assessments.

\section{Conclusion}

In conclusion, patients with SUD comorbidity are of greatest concern to referring teams. SUDs associated problems have also become the leading assessment and management issue for Australian general hospital CLP services when there is not a dedicated drug and alcohol liaison service. Patients with SUD comorbidity had distinct problems engaging in their treatment, housing situation, and psychiatric medication polypharmacy compared with patients with multiple non-SUD psychiatric disorders. Caution should be exercised in the interpretation of the multiple psychiatric diagnosis measure depending on the inclusion or exclusion of comorbid SUDs. CLP services should exercise a high degree of clinical suspicion for PTSD in patients with multiple substance use, as well as comorbidity with Cluster B personality disorders.

\section{Declarations}

\section{Acknowledgements}

We thank Dr StellaMay Gwini for assistance with the study design and statistical analysis and Professor Trisha Dunning for proofreading the article. 


\section{Funding}

This research did not receive any specific grant from funding agencies in the public, commercial, or notfor-profit sectors.

Competing interests: The authors declare no competing interests.

\section{References}

1. AlHW. (2012). Comorbidity of mental disorders and physical conditions 2007. Cat. no. PHE 155. Canberra: Australian Institute of Health and Welfare.

2. Bourgeois, J. A., Wegelin, J. A., Servis, M. E., \& Hales, R. E. (2005). Psychiatric Diagnoses of 901 Inpatients Seen by Consultation-Liaison Psychiatrists at an Academic Medical Center in a Managed Care Environment. Psychosomatics, 46, 47-57.

3. Buckley, P. F., \& Brown, E. S. (2006). Prevalence and consequences of dual diagnosis. J Clin Psychiatry, 67(7), e01.

4. Butler, K., Reeve, R., Viney, R., \& Burns, L. (2016). Estimating prevalence of drug and alcohol presentations to hospital emergency departments in NSW, Australia: impact of hospital consultation liaison services. Public Health Res Pract, 26(4).

5. Chandarana, P. C., Conlon, P., \& Steinberg, N. (1988). The evaluation of a consultation-liaison service. Gen Hosp Psychiatry, 10(5), 378-381.

6. Clarke, D. M., \& Smith, G. C. (1995). Consultation-liaison psychiatry in general medical units. Aust NZ J Psychiatry, 29(3), 424-432.

7. Desai, N. D., Shah, S. N., Shah, S. H., Sharma, E., \& Zankat, D. (2016). Patterns Of Psychiatric Referrals In Tertiary Care Hospital: An Overview Of Consultation Liaison Psychiatry. National Journal of Integrated Research in Medicine, 7(2), 56-60.

8. Devasagayam, D., \& Clarke, D. (2016). Evaluation of consultation-liaison psychiatry referrals from a critical care unit of an outer suburban hospital. Australas Psychiatry, 24(2), 168-172.

9. Diefenbacher, A., \& Strain, J. J. (2002). Consultation-liaison psychiatry: stability and change over a 10-year-period. Gen Hosp Psychiatry, 24(4), 249-256.

10. Diehl, A., Nakovics, H., Croissant, B., Reinhard, I., Kiefer, F., \& Mann, K. (2009). Consultation-liaison psychiatry in general hospitals: improvement in physicians' detection rates of alcohol use disorders. Psychosomatics, 50(6), 599-604.

11. Dilts, S. L., Jr., Mann, N., \& Dilts, J. G. (2003). Accuracy of referring psychiatric diagnosis on a consultation-liaison service. Psychosomatics, 44(5), 407-411.

12. Donald, F., Arunogiri, S., \& Lubman, D. I. (2019). Substance use and borderline personality disorder: fostering hope in the face of complexity. Australas Psychiatry, 27(6), 569-572. 
13. Holmes, A., Handrinos, D., Theologus, E., \& Salzberg, M. (2011). Service use in consultation-liaison psychiatry: guidelines for baseline staffing. Australas Psychiatry, 19(3), 254-258.

14. Huyse, F. J., Herzog, T., Lobo, A., Malt, U. F., Opmeer, B. C., Stein, B., et al. (2001). Consultation-Liaison psychiatric service delivery: results from a European study. Gen Hosp Psychiatry, 23(3), 124-132.

15. Khantzian, E. J. (1997). The self-medication hypothesis of substance use disorders: a reconsideration and recent applications. Harv Rev Psychiatry, 4(5), 231-244.

16. Krautgartner, M., Alexandrowicz, R., Benda, N., \& Wancata, J. (2006). Need and utilization of psychiatric consultation services among general hospital inpatients. Soc Psychiatry Psychiatr Epidemiol, 41(4), 294-301.

17. Lipowski, Z. J., \& Wolston, E. J. (1981). Liaison psychiatry: referral patterns and their stability over time. Am J Psychiatry, 138(12), 1608-1611.

18. Loewenstein, R. J., \& Sharfstein, S. S. (1983). Psychiatric consultations at the NIH. Gen Hosp Psychiatry, 5(2), 83-87.

19. Lubman, D. I., Hall, K., Pennay, A., \& Rao, S. (2011). Managing borderline personality disorder and substance use - an integrated approach. Aust Fam Physician, 40(6), 376-381.

20. Lyne, J., O'Donoghue, B., Bonnar, M., Golden, D., Mclnerney, C., Callanan, I., et al. (2010). Reasons for referral and consultation liaison psychiatry diagnoses. Ir J Psych Med, 27(3), 123-129.

21. McKegney, F. P., McMahon, T., \& King, J. (1983). The use of DSM-III in a general hospital consultationliaison service. Gen Hosp Psychiatry, 5(2), 115-121.

22. Mills, K. L., Teesson, M., Ross, J., \& Peters, L. (2006). Trauma, PTSD, and substance use disorders: findings from the Australian National Survey of Mental Health and Well-Being. Am J Psychiatry, 163(4), 652-658.

23. Nesse, R. M., \& Berridge, K. C. (1997). Psychoactive drug use in evolutionary perspective. Science, 278(5335), 63-66.

24. Nordeck, C. D., Welsh, C., Schwartz, R. P., Mitchell, S. G., Cohen, A., O'Grady, K. E., et al. (2018). Rehospitalization and substance use disorder (SUD) treatment entry among patients seen by a hospital SUD consultation-liaison service. Drug Alcohol Depend, 186, 23-28.

25. Pagura, J., Stein, M. B., Bolton, J. M., Cox, B. J., Grant, B., \& Sareen, J. (2010). Comorbidity of borderline personality disorder and posttraumatic stress disorder in the U.S. population. $J$ Psychiatr Res, 44(16), 1190-1198.

26. Regier, D. A., Farmer, M. E., Rae, D. S., Locke, B. Z., Keith, S. J., Judd, L. L., et al. (1990). Comorbidity of mental disorders with alcohol and other drug abuse. Results from the Epidemiologic Catchment Area (ECA) Study. JAMA, 264(19), 2511-2518.

27. Rothenhausler, H. B., Ehrentraut, S., \& Kapfhammer, H. P. (2001). Changes in patterns of psychiatric referral in a German general hospital: results of a comparison of two 1-year surveys 8 years apart. Gen Hosp Psychiatry, 23(4), 205-214. 
28. Simon, G. E., \& VonKorff, M. (1991). Somatization and psychiatric disorder in the NIMH Epidemiologic Catchment Area study. Am J Psychiatry, 148(11), 1494-1500.

29. Smith, G. C., Clarke, D. M., \& Handrinos, D. (1995). Recognising drug and alcohol problems in patients referred to consultation-liaison psychiatry. Med J Aust, 163(6), 307, 310 - 302.

30. Smith, G. C., Clarke, D. M., \& Herrman, H. E. (1993). Establishing a consultation-liaison psychiatry clinical database in an Australian general hospital. Gen Hosp Psychiatry, 15(4), 243-253.

31. Smothers, B. A., Yahr, H. T., \& Ruhl, C. E. (2004). Detection of alcohol use disorders in general hospital admissions in the United States. Arch Intern Med, 164(7), 749-756.

32. Tadros, G., Salama, R. A., Kingston, P., Mustafa, N., Johnson, E., \& Pannell, R.,

33. Hashmi, M. (2013). Impact of an integrated rapid response psychiatric liaison team on quality improvement and cost savings: the Birmingham RAID model. The Psychiatrist, 37, 4-10.

34. Tran, Q. N., Lambeth, L. G., Sanderson, K., de Graaff, B., Breslin, M., Huckerby, E. J., et al. (2020). Trend of emergency department presentations with a mental health diagnosis in Australia by diagnostic group, 2004-05 to 2016-17. Emerg Med Australas, 32(2), 190-201.

35. Trull, T. J., Freeman, L. K., Vebares, T. J., Choate, A. M., Helle, A. C., \& Wycoff, A. M. (2018). Borderline personality disorder and substance use disorders: an updated review. Borderline Personal Disord Emot Dysregul, 5, 15.

36. Wancata, J., Benda, N., Windhaber, J., \& Nowotny, M. (2001). Does psychiatric comorbidity increase the length of stay in general hospitals? Gen Hosp Psychiatry, 23(1), 8-14.

37. Wand, A. P. F., Wood, R., Macfarlane, M. D., \& Hunt, G. E. (2016). Comparison of consultation-liaison psychiatry services for inner-city, district or regional general hospitals using a common tool: Does one size fit all? J Psychosom Res, 84, 13-21.

38. Wapp, M., van de Glind, G., van Emmerik-van Oortmerssen, K., Dom, G., Verspreet, S., Carpentier, P. J., et al. (2015). Risk Factors for Borderline Personality Disorder in Treatment Seeking Patients with a Substance Use Disorder: An International Multicenter Study. Eur Addict Res, 21(4), 188-194.

39. Wood, R., Wand, A. P., \& Hunt, G. E. (2015). Relationship between timeliness of contact and length of stay in older and younger patients of a consultation-liaison psychiatry service. BJPsych Bull, 39(3), 128-133.

40. Zimmerman, M., \& Mattia, J. I. (1999). Psychiatric diagnosis in clinical practice: is comorbidity being missed? Compr Psychiatry, 40(3), 182-191.

\section{Figures}




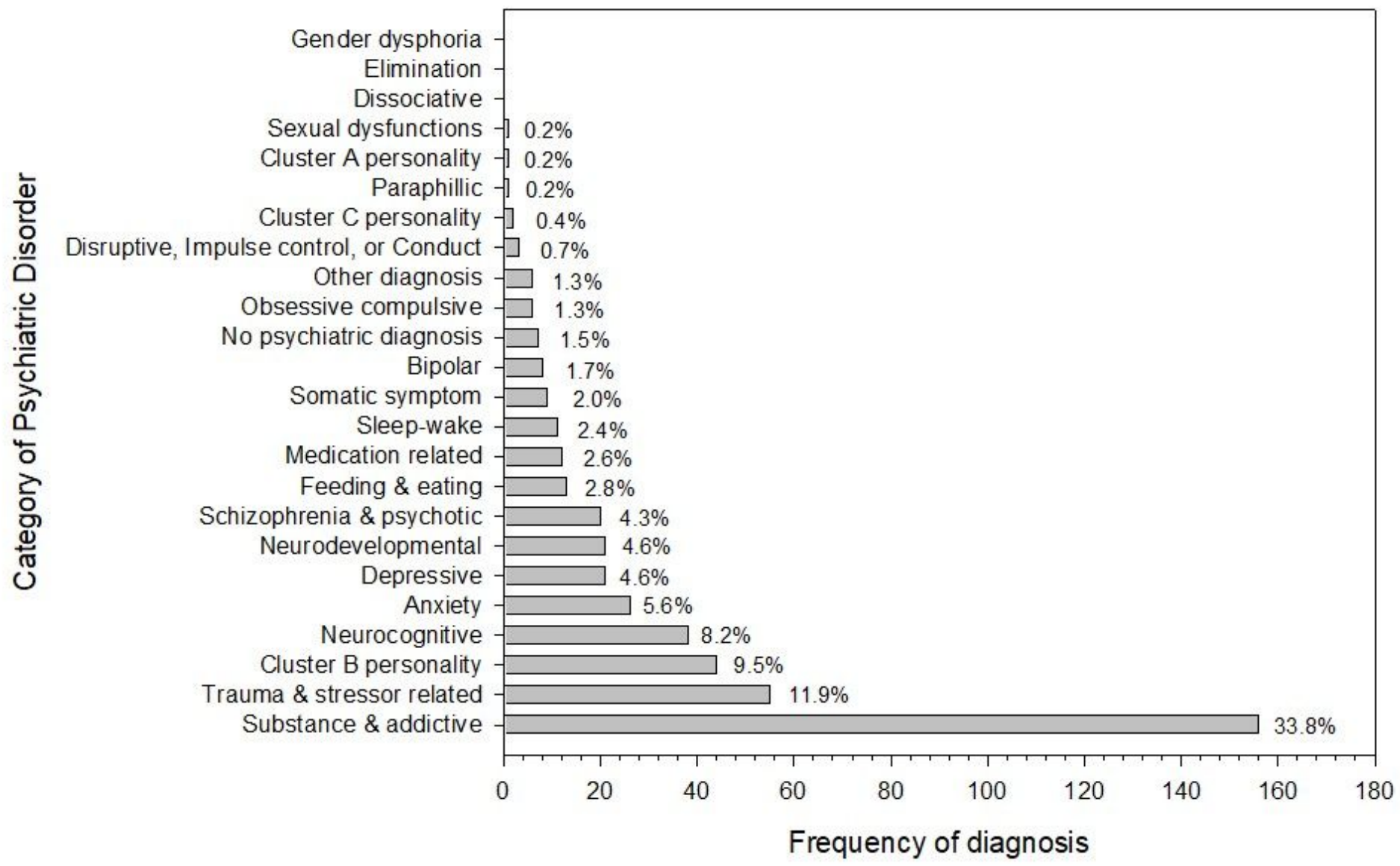

\section{Figure 1}

Figure 1. Diagnoses made by the CLP team according to DSM-5 categories. The percentage of total diagnoses is indicated next to each bar on the plot. 


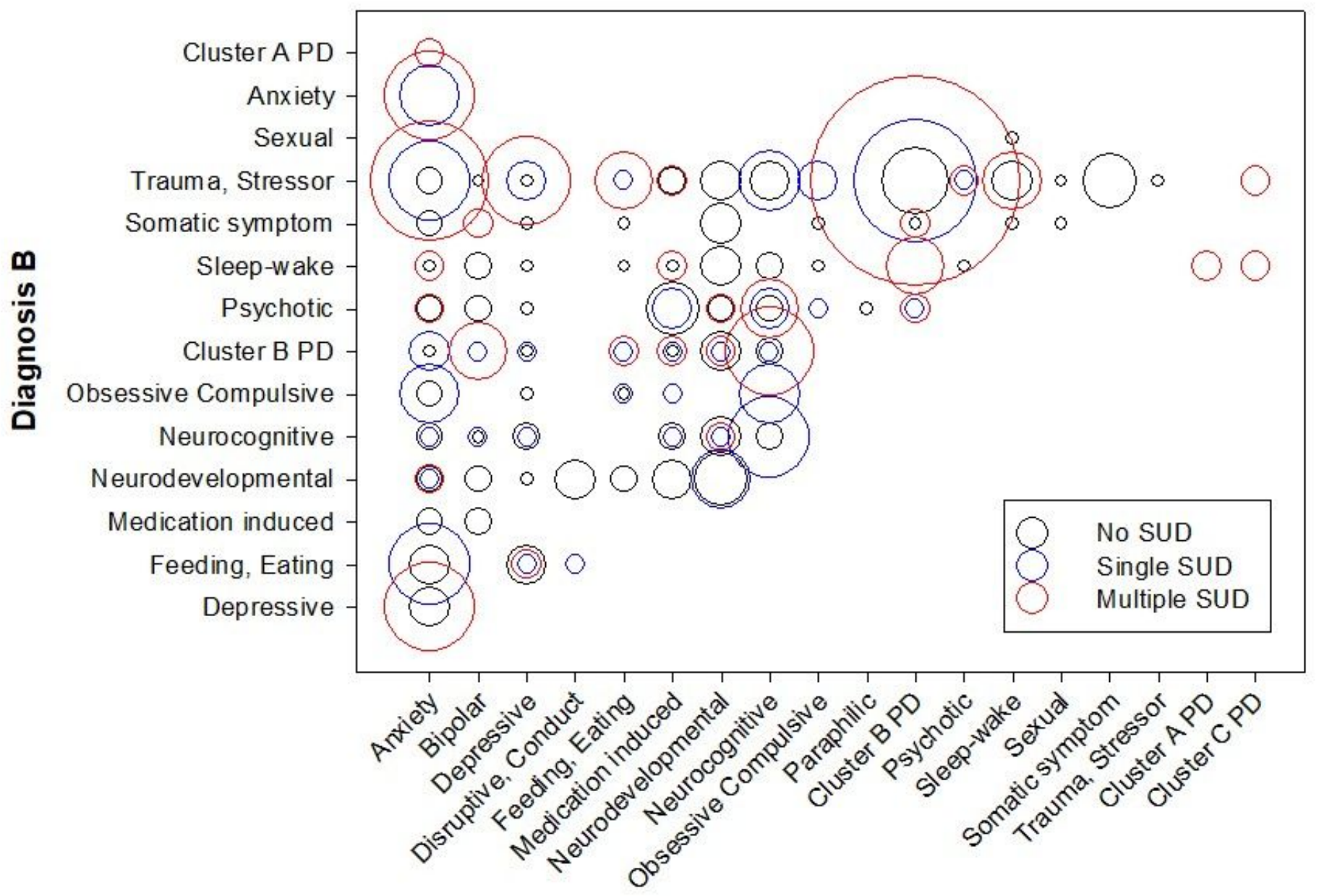

Diagnosis A

\section{Figure 2}

Figure 2. Bubble plot for co-occurrences of psychiatric diagnoses observed within the cohort of patients. The size of each bubble is proportionate to the observed frequency of each A-B diagnostic combination. Three series are plotted which represent patients with no substance use (black), single SUD comorbid with at least two other psychiatric diagnoses (blue), and multiple SUD comorbid with at least two other psychiatric diagnoses (red). Bubble sizes were normalized to number of patients within each group. 


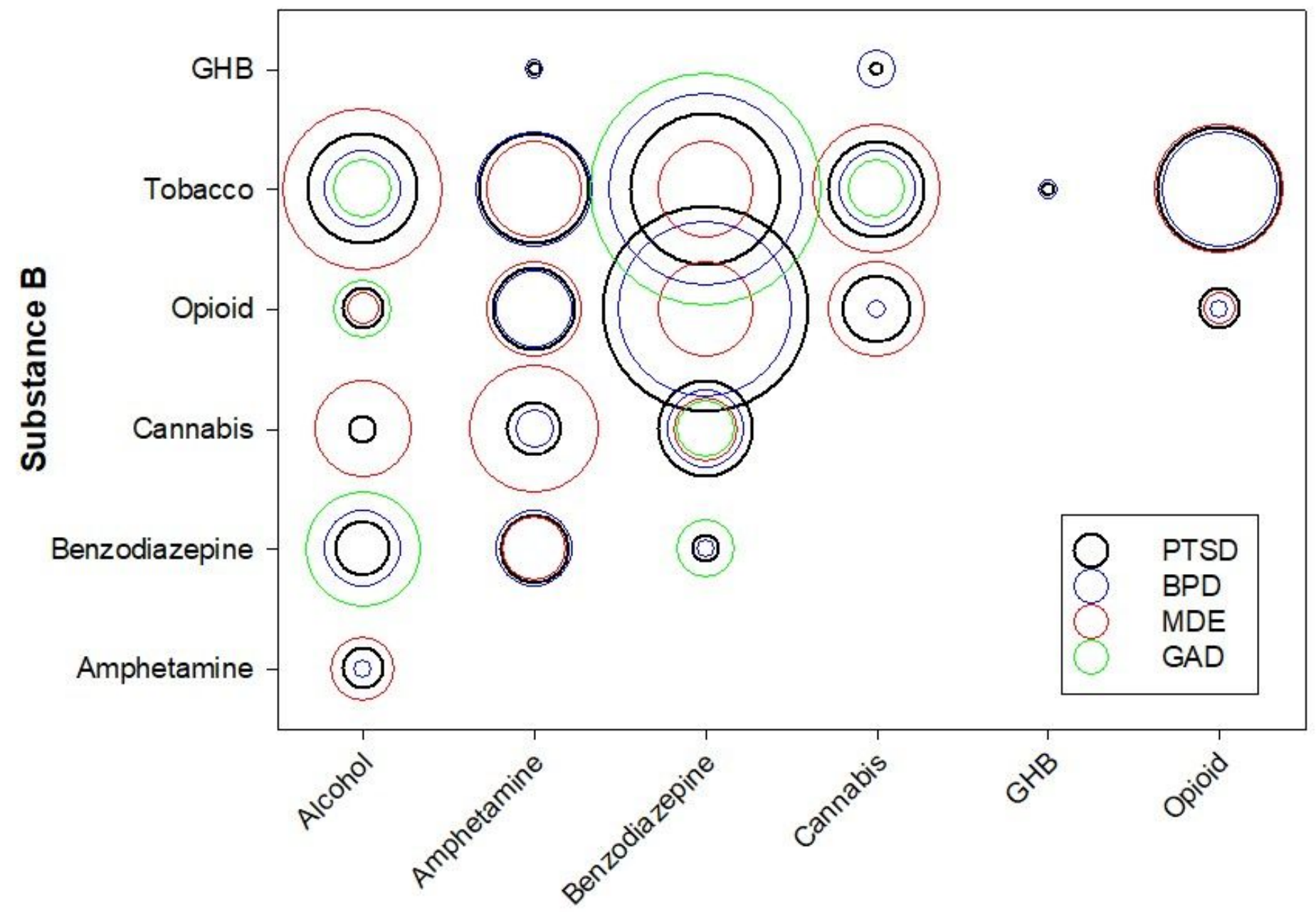

Substance A

\section{Figure 3}

Figure 3. Bubble plot for combinations of substances observed for patients who reported using at least two substances of dependence. The plots include both illicit and prescription substances of dependence. The size of each bubble is proportionate to the observed frequency of each A-B substance use combination. Separate series are plotted according to the commonest psychiatric diagnoses observed in the study. Bubble sizes were normalized to number of patients within each diagnostic group. 